\title{
Identification of key genes and regulators associated with carotenoid metabolism in apricot (Prunus armeniaca) fruit using weighted gene coexpression network analysis
}

Lina Zhang ${ }^{1 \dagger}$, Qiuyun Zhang ${ }^{2 \dagger}$, Wenhui $\mathrm{Li}^{3}$, Shikui Zhang ${ }^{3}$ and Wanpeng $\mathrm{Xi}^{{ }^{*}}$

\begin{abstract}
Background: Carotenoids are a class of terpenoid pigments that contribute to the color and nutritional value of many fruits. Their biosynthetic pathways have been well established in a number of plant species; however, many details of the regulatory mechanism controlling carotenoid metabolism remain to be elucidated. Apricot is one of the most carotenoidrich fruits, making it a valuable system for investigating carotenoid metabolism. The purpose of this study was to identify key genes and regulators associated with carotenoid metabolism in apricot fruit based on transcriptome sequencing.

Results: During fruit ripening in the apricot cultivar 'Luntaixiaobaixing' (LT), the total carotenoid content of the fruit decreased significantly, as did the levels of the carotenoids $\beta$-carotene, lutein and violaxanthin $(p<0.01)$. RNA sequencing (RNA-Seq) analysis of the fruit resulted in the identification of 44,754 unigenes and 6916 differentially expressed genes (DEGs) during ripening. Among these genes, 33,498 unigenes were annotated using public protein databases. Weighted gene coexpression network analysis (WGCNA) showed that two of the 13 identified modules ('blue' and 'turquoise') were highly correlated with carotenoid metabolism, and 33 structural genes from the carotenoid biosynthetic pathway were identified. Network visualization revealed 35 intramodular hub genes that putatively control carotenoid metabolism. The expression levels of these candidate genes were determined by quantitative real-time PCR analysis, which showed ripening-associated carotenoid accumulation. This analysis revealed that a range of genes (NCED1, CCD1/4, PIF3/4, HY5, ERF003/5/12, RAP2-12, AP2, AP2-like, BZR1, MADS14, NAC2/25, MYB1R1/44, GLK1/2 and WRKY6/31/69) potentially affect apricot carotenoid metabolism during ripening. Based on deciphering the molecular mechanism involved in ripening, a network model of carotenoid metabolism in apricot fruit was proposed.
\end{abstract}

Conclusions: Overall, our work provides new insights into the carotenoid metabolism of apricot and other species, which will facilitate future apricot functional studies and quality breeding through molecular design.

Keywords: Carotenoids, Apricot, Color, WGCNA, Prunus armeniaca L

\footnotetext{
* Correspondence: xwp1999@zju.edu.cn

${ }^{\dagger}$ Lina Zhang and Qiuyun Zhang contributed equally to this work.

${ }^{1}$ College of Horticulture and Landscape Architecture, Southwest University,

Chongqing 400716, People's Republic of China

Full list of author information is available at the end of the article
}

(c) The Author(s). 2019 Open Access This article is distributed under the terms of the Creative Commons Attribution 4.0 International License (http://creativecommons.org/licenses/by/4.0/), which permits unrestricted use, distribution, and reproduction in any medium, provided you give appropriate credit to the original author(s) and the source, provide a link to the Creative Commons license, and indicate if changes were made. The Creative Commons Public Domain Dedication waiver (http://creativecommons.org/publicdomain/zero/1.0/) applies to the data made available in this article, unless otherwise stated. 


\section{Background}

Carotenoids are widely distributed secondary metabolites that play important roles in plant physiology and are beneficial to human health as dietary components [1]. They confer flowers and fruits with yellow, orange and red colors, thereby helping to attract insects or animals to disperse seeds and pollen, and they are involved in photosynthesis and photoprotection. Carotenoids also scavenge free radicals in plants and help resist biotic and abiotic stresses [2]. In humans, dietary carotenoids provide vitamin A precursors, scavenge free radicals, strengthen immunity and prevent various diseases, such as certain cancers and cardiovascular diseases [3].

The carotenoid biosynthesis pathway has been well characterized in many plants [4]. The process starts with geranylgeranyl diphosphate (GGPP) synthesis, which involves a condensation reaction between isopentenyl diphosphate isomerase (IPP) and dimethylallyl diphosphate (DMAPP). GGPP is then converted into phytoene by phytoene synthase (PSY), which is in turn converted into lycopene via a series of desaturation and isomerization steps. Lycopene is cyclized by $\varepsilon$-cyclase (LCYE) and $\beta$-cyclase (LCYB) or by LCYB to produce $\alpha$-carotene or $\beta$-carotene, respectively. These carotenes are further hydroxylated to produce zeaxanthin. Carotenoids can be cleaved into apocarotenoids (e.g., $\beta$-ionone, strigolactones, $\beta$-citraurin and abscisic acid) by carotenoid cleavage dioxygenase (CCD) and 9-cisepoxycarotenoid dioxygenase (NCED) [5-7]. The associated structural genes in this biosynthetic pathway have been studied in detail in a number of plant species [1]. In tomato, the increased production of lycopene during the ripeningrelated fruit color change from green to red is caused by increased transcription of genes from early 'upstream' steps in the biosynthetic pathway, including PSY, phytoene desaturase $(P D S)$, carotenoid isomerase (CRTISO) and deoxy-dxylulose-5-phosphate synthase $(D X S)$, and the downregulation of the 'downstream genes' $L C Y B, L C Y E$, and $\beta$ carotene hydroxylase $(C H Y B)$ [8-11]. Additionally, several transcription factors associated with carotenoid accumulation have been identified. For example, ethylene response factors (ERFs) and phytochrome interacting factors (PIFs) have been shown to reduce carotenoid accumulation by binding specifically to the AtPSY and AtPDS promoter in Arabidopsis thaliana [12, 13]. In tomato fruit tissues, the MADS box transcription factor ripening inhibitor (RIN) has been reported to regulate carotenoid accumulation by interacting with the SIPSY1 promoter [14, 15]. However, many details of the regulatory network controlling carotenoid metabolism have yet to be identified.

Apricot (Prunus armeniaca L.) is an important fruit crop that is grown in temperate climates. Fresh apricots are an excellent source of diverse nutrients, including carotenoids, polyphenols, ascorbic acid and various microelements [16]. Processed apricot fruit byproducts have also been suggested to be valuable for human nutrition and the treatment of different diseases [16]. The 'Luntaixiaobaixing' (LT) apricot cultivar from China is commercially popular and valued by consumers due to its attractive flavor, shape and color. During ripening, its color changes from green to yellow and then to light yellow, and the fruit exhibits a reduction in carotenoid levels [17], which involves a range of carotenoid metabolic reactions, including synthesis, accumulation and cleavage. Thus, the 'LT' cultivar represents an excellent model for studying the regulatory mechanism of carotenoid metabolism.

RNA sequencing (RNA-Seq) is a powerful technology for studying gene expression, identifying genes and performing functional analysis [18], due to its high throughput, low cost, high accuracy and high sensitivity [19]. In recent years, RNA-Seq has been applied to the study of many fruit species to characterize the molecular processes underlying ripening and secondary metabolism [20-23]. It is a particularly effective approach for species for which whole-genome sequence data are not yet available, such as apricot. At present, the public resources of apricot transcriptome data are still very limited [24, 25].

In this study, we integrated weighted gene coexpression network analysis (WGCNA), gene-trait correlations and differential expression analysis in apricot fruit to identify key genes and regulators related to carotenoid metabolism during fruit ripening. Based on this analysis, a network model of carotenoid metabolism is proposed. These data will help advance the functional genomic analysis of carotenoid metabolism in apricot and other species.

\section{Results}

\section{Changes in basic quality parameters and carotenoid} content during apricot fruit ripening

During the three apricot ripening stages ( $\mathrm{T}$, turning, 57 DPA (days post anthesis); CM, commercial maturation, 65 DPA; FR, fully ripe, 74 DPA), 'LT' fruit color changed from green to yellow and then from yellow to light yellow (Fig. 1a). Over the same period, fruit firmness dramatically decreased from $25.55 \mathrm{~N}$ to $2.77 \mathrm{~N}$ compression $(p<0.01)$, but fruit weight sharply increased from an average of $8.7 \mathrm{~g}$ to $19.0 \mathrm{~g}$ (Fig. 1b). Similarly, the content of fruit total soluble solids (TSS) significantly increased from $10.1^{\circ}$ Brix to $19.9^{\circ}$ Brix, while titratable acidity (TA) decreased substantially from $2.43 \mathrm{mg} / \mathrm{mL}$ to $0.4 \mathrm{mg} / \mathrm{mL}(p<0.01)$ (Fig. 1b). We also observed that the citrus color index $(\mathrm{CCI})$ values of the fruit significantly increased from -6.77 to -0.64 during ripening $(p<0.01)$ (Fig. 1c). Notably, the levels of lutein, violaxanthin and $\beta$-carotene significantly decreased during ripening (Fig. 1c), and the total carotenoid content markedly decreased from $51.4 \mu \mathrm{g} / \mathrm{g}$ fresh weight (FW) to $21.3 \mu \mathrm{g} / \mathrm{g}$ FW $(p<0.01)$. Conversely, the contents of neoxanthin and phytoene increased during the process (Fig. 1c). These results indicated that the 'LT' fruit underwent normal ripening based on a range of physiological and 


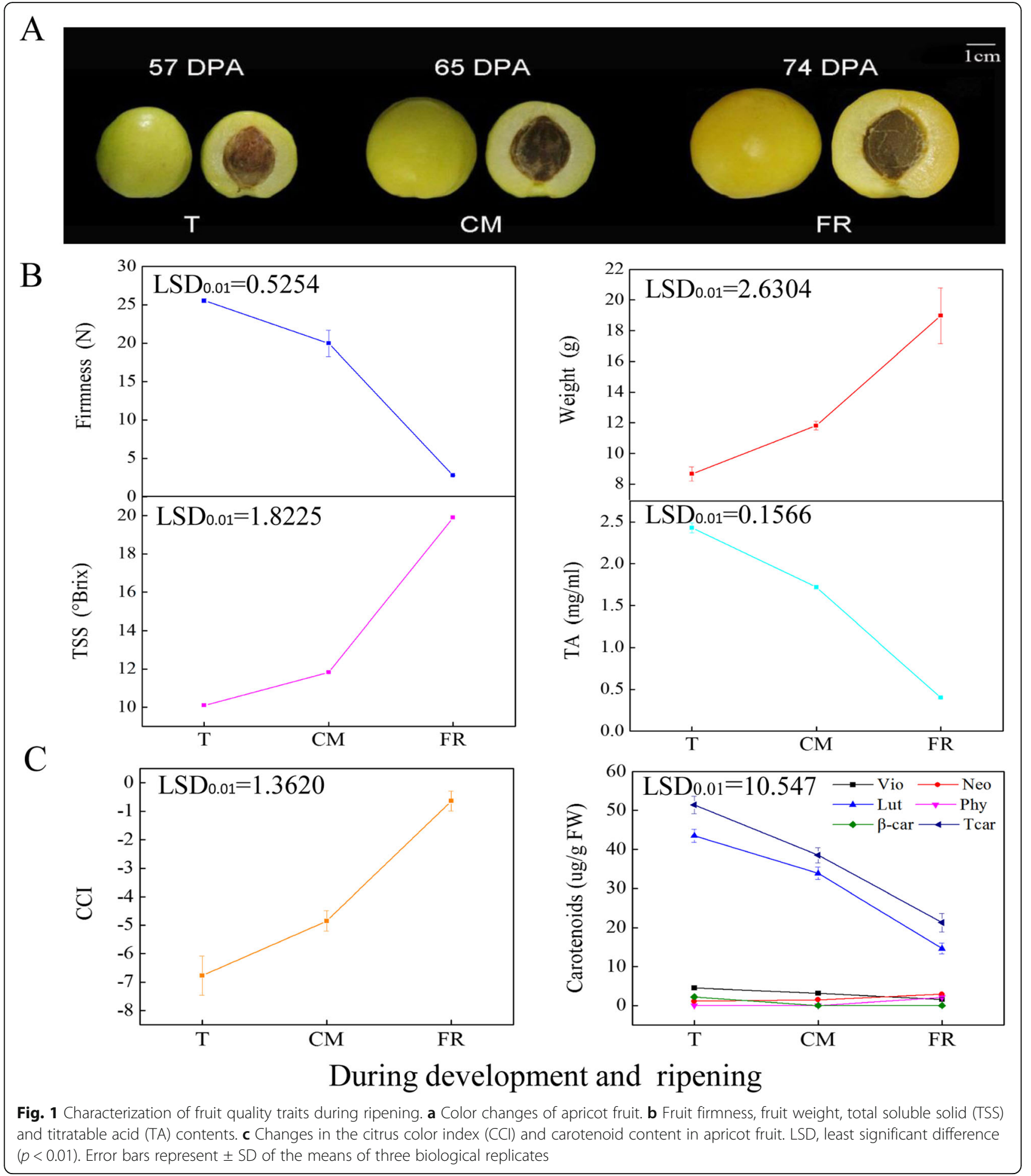

biochemical characteristics and that the cultivar is a 'carotenoid loss' type of cultivar.

\section{Transcriptome analysis of fruit ripening}

Nine mRNA libraries were generated from the $T, C M$ and FR stages of 'LT' fruit, with three biological replicates per stage. Average clean read numbers of 26.3, 26.0 and 27.2 million were derived from the T, CM and FR stages, respectively, corresponding to approximately 2.37, 2.34 and 2.45 gigabase pairs of nucleotides (nt), with GC percentages of $45.88,45.58$ and $46.16 \%$ (Additional file 1). Principal component analysis (PCA) 
confirmed the reproducibility of the sequence data for each stage (Fig. 2).

Next, a total of 44,754 unigenes with a mean length of $1196 \mathrm{bp}$ and an N50 length of $1860 \mathrm{nt}$ and a total of 71,243 contigs with a mean length of $529 \mathrm{bp}$ and an N50 of 1303 nt, were assembled using Trinity software (Additional file 2). A total of 23,784 unigenes were assigned gene ontology (GO) terms and classified into 55 subcategories within the three standard categories of 'biological process', 'cellular component' and 'molecular function'. The subcategories 'metabolic process' and 'cellular process' were the most highly enriched in the 'biological process' domain, while 'cell' and 'cell part' were the most highly enriched subcategories in the 'cellular component' domain, and 'catalytic activity' and 'binding' were the top ranked terms in the 'molecular function' domain (Additional file 3).

\section{Identification of differentially expressed genes (DEGs)}

On the basis of the mapped reads, a total of 6916 DEGs were identified using fragments per kilobase of transcript per million mapped reads (FPKM) values, with 2436, 4801 and 5728 DEGs present in the T, CM and FR
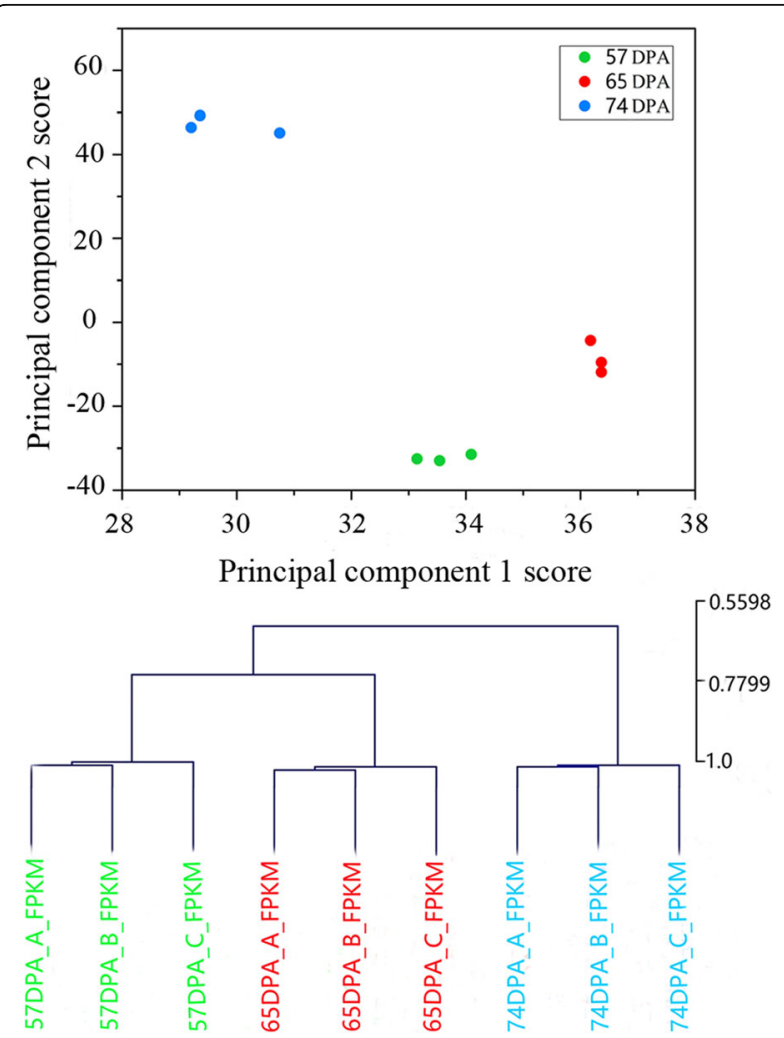

Fig. 2 Principal component analysis (PCA) of transcriptome data. Three biological replicates per sample were analyzed for each ripening stage. The percentages on the axes indicate the values explained by each PCA. The green, red, and sky blue dots represent the transcriptomes of the samples obtained at 57 DPA (days post anthesis), 65 DPA and 74 DPA, respectively stages, respectively (Fig. 3a). The numbers of unique DEGs in the different stages were 630, 1288, and 1561 at 57 vs. 65 DPA, 65 vs .74 DPA and 57 vs. 74 DPA, respectively, and a total of 448 genes were expressed at all three stages. The identified DEGs were selected for further analysis (Fig. 3b and Additional file 4).

\section{GO enrichment and Kyoto encyclopedia of genes and genomes (KEGG) pathway analysis}

To help identify the biological functions of the DEGs, the GO and KEGG databases were used to identify enriched terms/pathways at the three ripening stages for the identified DEGs. 'Cellular process', 'metabolic process' and 'single organism process' were the main categories in the 'biological process' domain, while the 'cell', 'cell part' and 'organelle' categories were enriched in the 'cellular component' domain. Finally, molecular functions such as 'binding', 'catalytic activity' and 'transporter activity' were the most highly enriched during the three ripening stages (Additional file 5).

KEGG pathway enrichment analysis was carried out to assess the biological significance of the DEGs during the three ripening stages. A total of 1773, 4719, 3215 unigenes from the T, CM and FR stages, respectively, were mapped to 20 KEGG pathways. 'Metabolic pathways', 'plant-pathogen interaction' and 'plant hormone signal transduction' were the most significantly enriched metabolic pathways. We identified 19, 33 and 16 DEGs from the carotenoid biosynthetic pathway enriched in the T, CM and FR stages, respectively. This analysis suggested that the three enriched metabolic pathways may play a role in carotenoid metabolism in apricot fruit (Additional file 6).

\section{Weighted gene coexpression network analysis (WGCNA)}

WGCNA is a method for describing the correlations in patterns of gene expression and for revealing clusters, or modules, of genes whose expression is highly correlated. This method allows the identification of module eigengenes and intramodular hub genes and the association of modules with one another and with sample traits [26]. Here, 13 coexpression modules were identified by WGCNA, among which the 'blue' and 'turquoise' modules were significantly associated with carotenoid metabolism during ripening (Fig. 4). Analysis of the moduletrait relationships suggested that the 'blue' module, containing 4981 genes, was highly positively correlated with levels of violaxanthin $(r=0.99, P=8 \mathrm{e}-07)$, lutein $(r=$ 0.84, $P=0.005)$ and $\beta$-carotene $(r=0.94, P=2 \mathrm{e}-04)$ and negatively correlated with neoxanthin $(r=-0.47, P=0.2)$ and phytoene $(r=-0.75, P=0.02)$ contents during ripening. The abundance of phytoene was highly positively correlated with gene expression in the 'turquoise' module, which contained 5258 genes, with a coefficient of $0.95(P=7 \mathrm{e}-05)$, but the levels of violaxanthin and lutein 


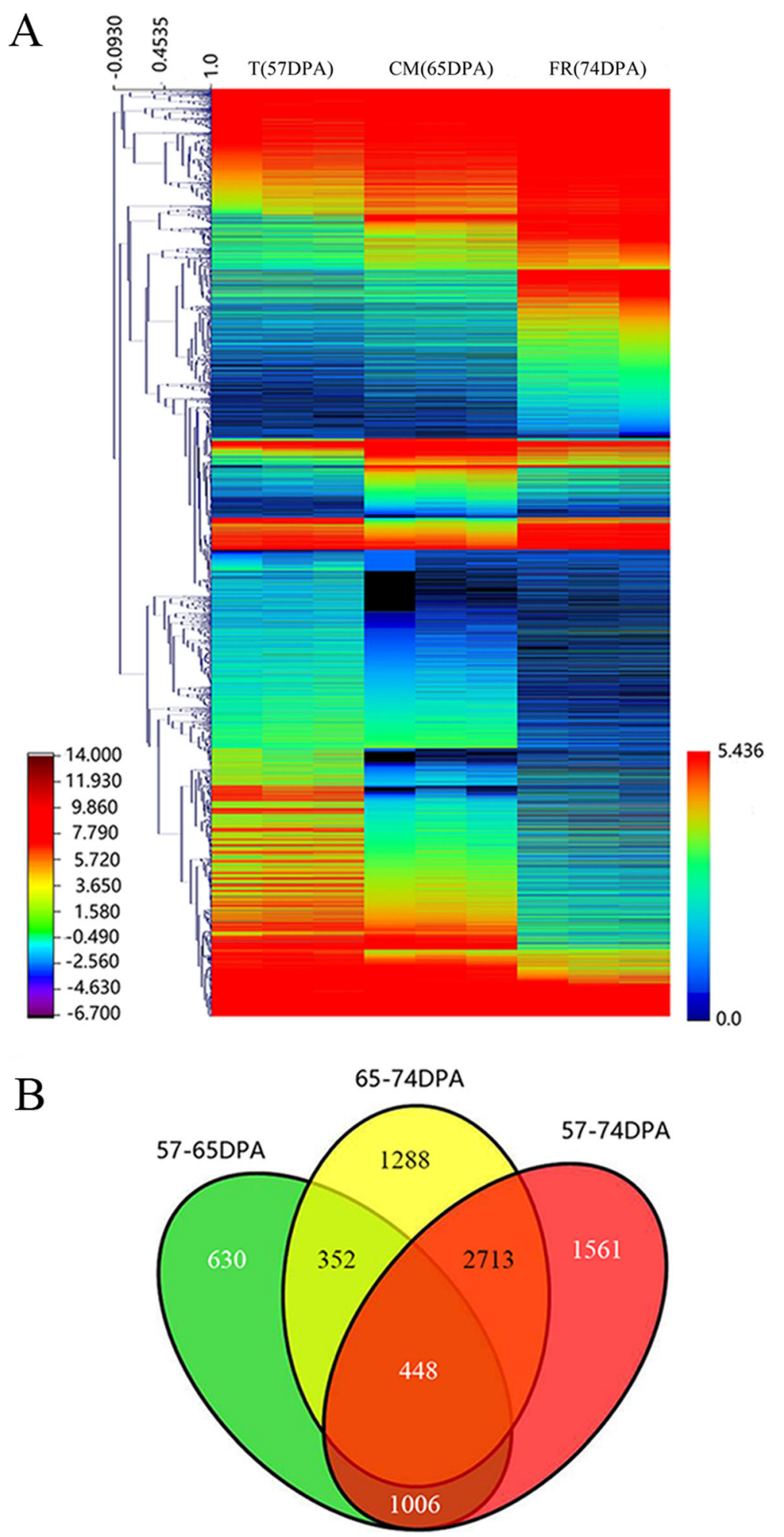

Fig. 3 Expression profiles of differentially expressed genes (DEGs) during apricot fruit ripening. a DEG expression dynamics during fruit ripening. b Venn diagram indicates the number of DEGs in each of the three ripening stages 


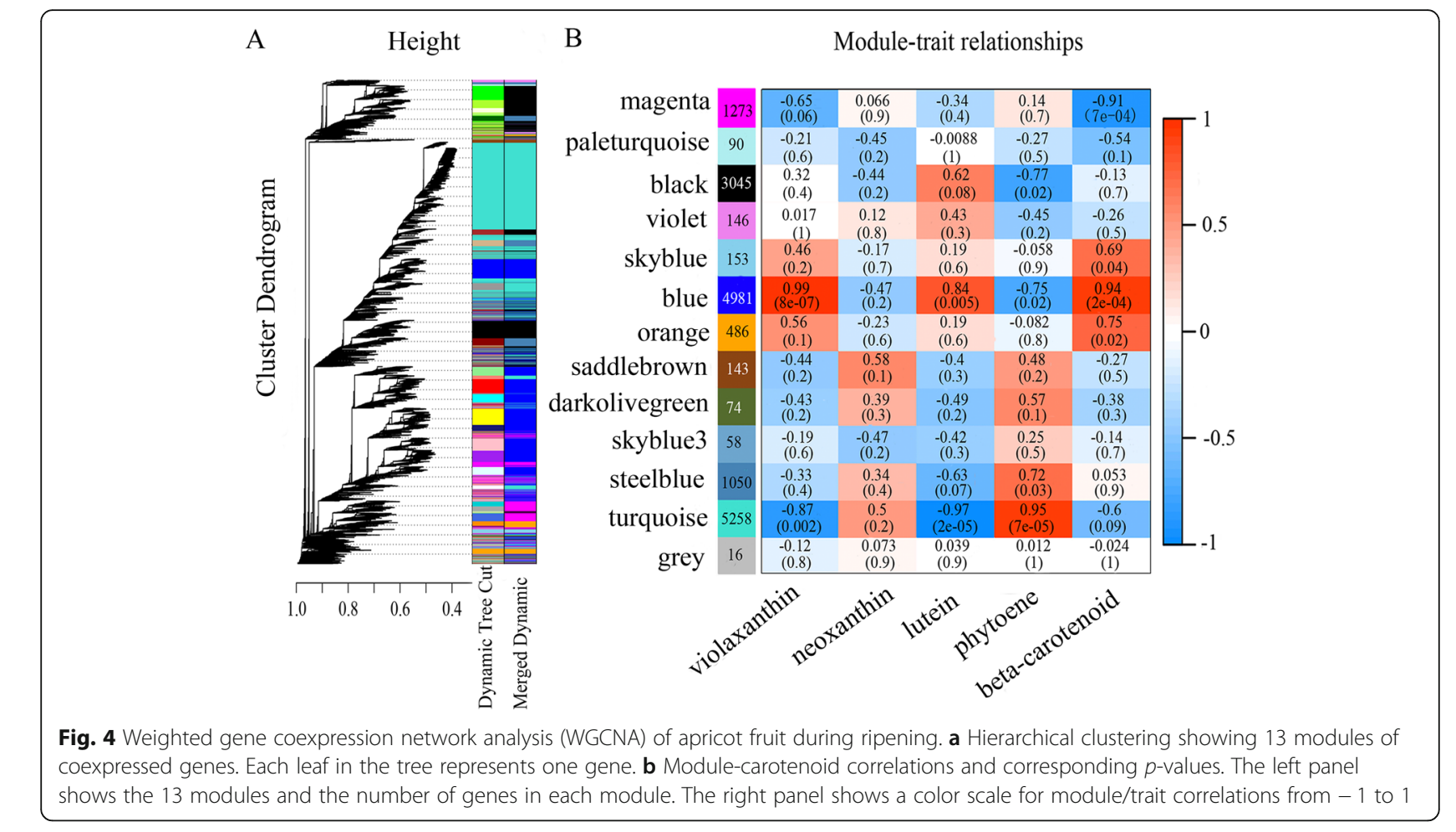

B Module-trait relationships

were significantly negatively correlated with gene expression, with coefficients of $-0.87(P=0.002)$ and -0.97 $(P=2 \mathrm{e}-05)$, respectively (Fig. $4 \mathrm{~b})$. These results suggested that the genes in these two modules were associated with carotenoid accumulation.

\section{DEGs involved in carotenoid biosynthesis}

In the carotenoid biosynthesis pathway (Fig. 5a), we identified 33 DEGs that were annotated as functioning in carotenoid metabolism; their expression levels were represented by both FPKM and qPCR values (Fig. 5b, Additional file 7), and good linear positive correlations were observed between them (Fig. 5c). During ripening, the expression levels of two IPI genes (U7591 and U10599) were downregulated and upregulated, respectively. In addition, the expression of the geranylgeranyl diphosphate synthase (GGPPS) gene was significantly upregulated, and the expression levels of two PSY (U14684 and U3487) genes were upregulated and up/ downregulated, respectively. The expression levels of four PDS1 genes gradually increased during ripening, with PDS1 (U17285) representing the exception. Three $\zeta$-carotene desaturase (ZDS) genes (CL1821.1, CL3007.2 and U15347) displayed similar up/downregulated expression patterns, and other $Z D S$ genes showed significant upregulation. CRTISO expression markedly decreased during ripening, and the expression of three $L C Y B$ genes showed similarly upregulated expression, with $L Y C B$ (CL4657.1) representing the exception, but the expression of two
$C H Y B$ genes showed the opposite expression pattern. Five zeaxanthin epoxidase $(Z E P)$ genes exhibited different levels of expression. Two violaxanthinde-epoxidase 1 (VDE1) genes were downregulated, while the expression of neoxanthin synthase $(N X S)$ was upregulated at the FR stage. The expression of the NCED1 gene as well as CCD1 and $C C D 4$ gradually increased during ripening. Thus, numerous DEGs that were involved in the ripening-related synthesis and accumulation of carotenoids were identified.

\section{Visualization of gene networks}

A total of 78 genes in the 'blue' module and 15 genes in the 'turquoise' module were analyzed using Cytoscape version 2.8. Both modules exhibited a significant association with carotenoid contents and gene expression of $>$ 0.4 (Fig. 6 and Additional file 8). In the two networks, 15 $E R F, 9 b H L H$ and 24 WRKY family members were identified as intramodular hub genes and were therefore putatively associated with the regulation of carotenoid metabolism. The carotenoid biosynthesis pathway genes PDS1, CCD1, CCD4, VDE1 and ZEP were identified as key control points for carotenoid metabolism (Fig. 6a and b). In addition to these structural genes, we identified transcription factors in the two modules, including U7919, C4195.1 and U6922, which are homologs of the Arabidopsis thaliana MYB1R1, MYB21 and MYB44 proteins, respectively, involved in the regulation of carotenoid metabolism. Homologs of the A. thaliana transcription factors MYC2, GLK1, GLK2, NAC25, HY5, 


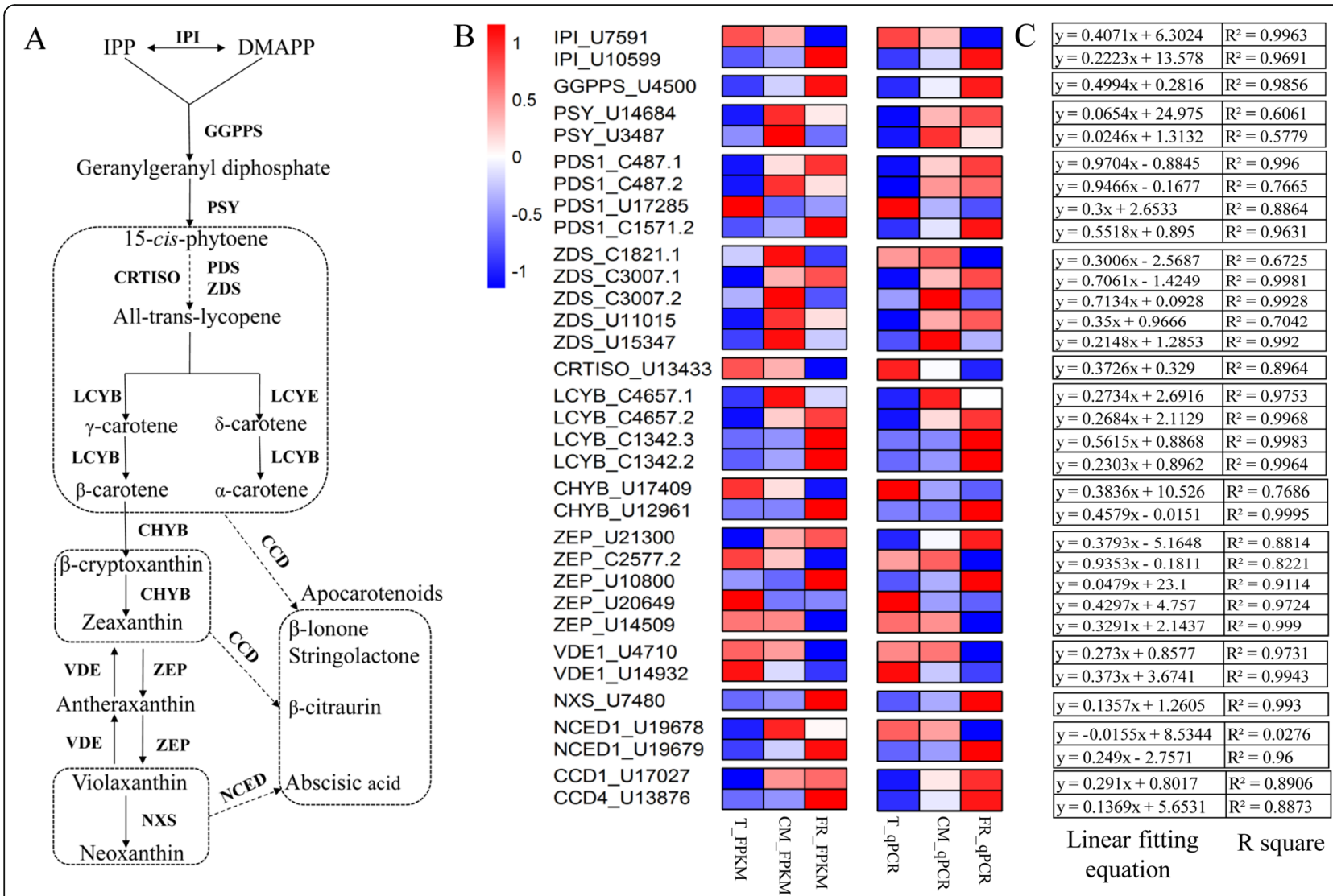

Fig. 5 Expression of genes related to carotenoid biosynthesis in apricot fruit. a Carotenoid biosynthesis pathway in apricot fruit. IPI, isopentenyl

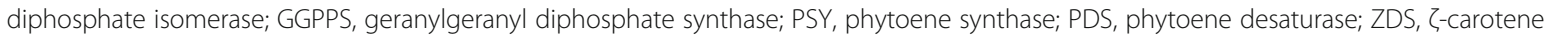
desaturase; CRTISO, carotenoid isomerase; LCYE, lycopene $\varepsilon$-cyclase; LCYB, lycopene $\beta$-cyclase; CHYB, $\beta$-carotene hydroxylase; ZEP, zeaxanthin epoxidase; VDE, violaxanthin de-epoxidase; NXS, neoxanthin synthase; CCD, carotenoid cleavage dioxygenase; NCED, 9-cis-epoxycarotenoid dioxygenase. $\mathbf{b}$ Heatmap of the expression of genes related to carotenoid biosynthesis during fruit ripening. Columns and rows represent samples and gene names in the heatmap, respectively. Red, blue and white indicate high expression, low expression and the absence (or undetectable levels) of detectable transcripts at the corresponding stage, respectively. c The linear fitting equation and $\mathrm{R}$ squared values between the FPKM and qRT-PCR values

PIF3, MADS14, WRKY6, WRKY31, WRKY69, ERF003, RAP2-12 (ERF), NAC and bHLH68 were also identified.

\section{Expression of candidate genes and regulators during fruit ripening}

To verify the reliability of the RNA-Seq results and ascertain the relationship between candidate genes and carotenoid metabolism, we also measured the expression levels of the 12 candidate structural genes and 35 transcription factors by qRT-PCR during fruit ripening (Fig. 7a). Overall, all upstream genes related to carotenoid biosynthesis, such as PDS1 (CL1571.2), ZEP (U21300 and U10800), CCD1/4 and capsanthin/capsorubin synthase $(C C S)$, were upregulated in the 'LT' fruit, while the expression levels of 15-cis-zeta-carotene isomerase ( $Z$ ISO), PDS1 (U17285), ZEP (U20649) and VDE1 were downregulated. ERF003, AP2-like, RAP2-12, NAC (CL3330.2), WRKY6/31 and BZR1 were significantly upregulated during ripening, but other genes showed opposite expression patterns. Linear regression analysis demonstrated that the FPKM values were highly correlated with the qRT-PCR results (Fig. 7b).

\section{Discussion}

An increasing number of studies highlight the complexity and diversity of carotenoid metabolism in different plant species [1, 4]. Here, we used the apricot cultivar 'LT', which exhibits a specific carotenoid accumulation pattern that includes biosynthesis, sequestration and degradation during fruit ripening, to identify key candidate genes and regulators related to carotenoid metabolism by WGCNA. Based on our results, we conclude that carotenoid metabolism in apricot fruit is cocontrolled by light signals, phytohormones, and developmental factors, and a network regulatory model was proposed (Fig. 8).

Light is a key factor in plant growth and development, since it provides energy for photosynthesis and regulates photomorphogenesis as a signal $[4,5]$. PIF is a member 


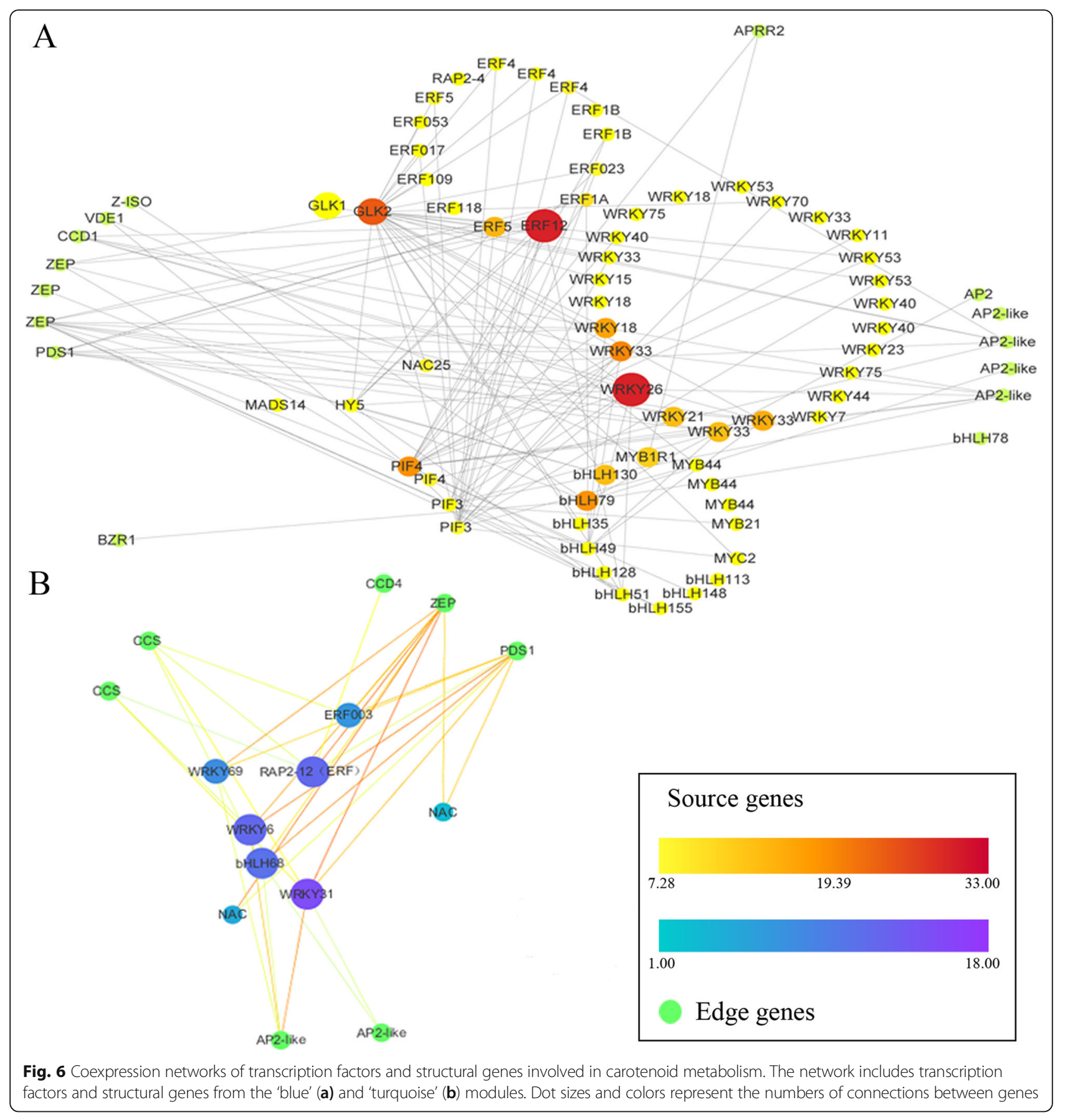

of the bHLH transcription factor family and can integrate multiple environmental and intercellular signals to control growth and development, stress responses and secondary metabolism. A light signal causes a rapid transfer of active phytochrome to the nucleus, where it interacts with PIFs $[27,28]$. In the present study, we observed that the expression of two PIF3 genes and two PIF4 genes was positively correlated with carotenoid accumulation during ripening (Fig. 1c and Fig. 7a). Our results are in agreement with the observation that PIF5 functions as a positive regulator of the MEP pathway and increases the accumulation of chlorophylls and carotenoids in cultured cells [29], but they contrast with the finding that PIF1 proteins bind directly to the G-box element in the PSY promoter to inhibit its expression, resulting in reduced carotenoid accumulation [13], which suggests that PIF gene members may play a different role in carotenoid metabolism. Two light signaling transcription factors, LONG HYPOCOTYL5 (HY5) and COP1-like, were previously identified in tomato fruit. In contrast to LeCOP1LIKE, repression of LeHY5 results 


\section{A 1 1 Z-ISO_U13433 PDS1_17285 PDS1_CL1571.2 0.5 ZEP U21300 ZEP U10800 0 ZEP_CL2577.2 ZEP U20649 \\ -0.5 VDE1_U14932 CCD1 U10727 CCD4 U13876 \\ $-1 \quad$ CCS CL1342.2 CCS_CL1342.3}

ERF1A_U10066 ERF003 U9624

ERF5 U19037

ERF12_U4802

AP2 CL1173.2

AP2-like_CL399.1

AP2-like_CL399.5

APRR2 U18810

RAP2-12_U16974

GLK1_U15452

GLK2_U15451

NAC25_CL2500.3

NAC U12188

NAC_CL3330.2

HY5_U5990

MADS14_U4096

PIF3 CL3263.2

PIF3 CL3263.1

PIF4 U14478

PIF4 CL4392.3

WRKY6 CL3232.2

WRKY18 CL402.4

WRKY21_U22295

WRKY26_U17411

WRKY31_U6036

WRKY33_CL2895.2

WRKY33 CL1588.5

WRKY33_CL1588.

WRKY69_U17748

bHLH68 U12776

bHLH79 CL184.1

bHLH130_CL128.9

MYB1R1 U7919

MYB44_CL1116.3

BZR1_U9500
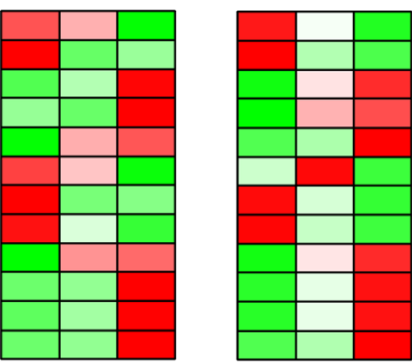

\begin{tabular}{ll|l|}
\hline$y=0.3893 x+1.3363$ & $R^{2}=0.8819$ \\
\hline
\end{tabular}

$y=0.3 x+2.6533$

\begin{tabular}{|l|l|}
\hline$y=0.3 x+2.6534 x+1.8999$ & $R^{2}=0.8471$ \\
\hline$y=0.0479 x+23.1$ & $R^{2}=0.914$ \\
\hline
\end{tabular}

\begin{tabular}{ll}
$\mathrm{y}=0.0479 \mathrm{x}+23.1$ & $\mathrm{R}^{2}=0.9114$ \\
\hline $\mathrm{y}=0.7445 \mathrm{x}-5.6678$ & $\mathrm{R}^{2}=0.9993$ \\
\hline
\end{tabular}

\begin{tabular}{|l|l|}
\hline$y=0.04945 x-5.6678$ & $R^{2}=0.9993$ \\
\hline$y=0.935 x-0.181 I$ & $R^{2}=0.82 I$ \\
\hline
\end{tabular}

\begin{tabular}{|l|l|}
\hline$y=0.9353 x-0.1811$ & $R^{2}=0.8221$ \\
\hline$y=0.7834 x-30752$ & $R^{2}=0.859$ \\
\hline
\end{tabular}

\begin{tabular}{ll}
$y=0.7834 x-3.0752$ & $R^{2}=0.8549$ \\
\hline$y=0.373 x+3.6741$ & $R^{2}=0.994$ \\
\hline
\end{tabular}

\begin{tabular}{ll}
$\mathrm{y}=0.373 \mathrm{x}+3.6741$ & $\mathrm{R}^{2}=0.9943$ \\
\hline$y=0.291 \mathrm{x}+0.7954$ & $\mathrm{R}^{2}=0.890$ \\
\hline
\end{tabular}

\begin{tabular}{|l|l|}
\hline$y=0.373 x+3.6741$ & $R^{2}=0.9943$ \\
\hline$y=0.2911 x+0.7954$ & $R^{2}=0.8909$ \\
\hline$y=0.1369 x+5.6531$ & $R^{2}=0.883$ \\
\hline
\end{tabular}

\begin{tabular}{ll}
$y=0.1369 x+5.6531$ & $R^{2}=0.8873$ \\
\hline$y=0.342 x+1.001$ & $R^{2}=0.925$ \\
\hline
\end{tabular}

\begin{tabular}{ll}
\hline$y=0.3424 x+1.001$ & $R^{2}=0.9254$ \\
\hline$y=0.288 x+0.8091$ & $2^{2}=0.9855$ \\
\hline
\end{tabular}

\begin{tabular}{|l|l|}
\hline$y=0.2988 x+0.8091$ & $R^{2}=0.9835$ \\
\hline
\end{tabular}
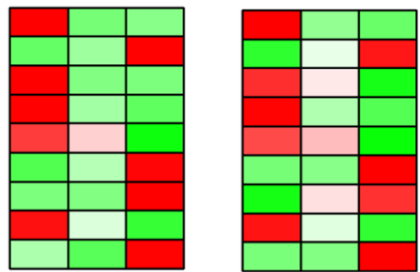

\begin{tabular}{|l|l|}
\hline $\mathrm{y}=0.1241 \mathrm{x}+2.9573$ & $\mathrm{R}^{2}=0.9822$ \\
\hline $\mathrm{y}=0.3349 \mathrm{x}+0.8761$ & $\mathrm{R}^{2}=0.91$ \\
\hline
\end{tabular}

\begin{tabular}{|l|l|}
\hline$y=0.124 x+2.9573$ & $R^{2}=0.9822$ \\
\hline$y=0.019 x+0.8761$ & $R^{2}=0.911$ \\
\hline
\end{tabular}

\begin{tabular}{|l|l|}
\hline$y=0.0195 x+7.8427$ & $R^{2}=0.6824$ \\
\hline$y=0.1003 x+1.499$ & $R^{2}=0.9974$ \\
\hline
\end{tabular}

\begin{tabular}{|l|l|}
\hline$y=0.1003 x+1.4699$ & $R^{2}=0.9974$ \\
\hline$y=0.5449 x-8.5573$ & $R^{2}=0.9932$ \\
\hline
\end{tabular}

\begin{tabular}{ll}
\hline $\mathrm{y}=0.5449 \mathrm{x}-8.5573$ & $\mathrm{R}^{2}=0.9932$ \\
\hline $\mathrm{y}=0.9129 \mathrm{x}+0.1533$ & $\mathrm{R}^{2}=0.9638$ \\
\hline
\end{tabular}

\begin{tabular}{|l|l|}
\hline$y=2.9129 x+0.1533$ & $R^{2}=0.9638$ \\
\hline$y=0.2351 x+0.7382$ & $R^{2}=0.6513$ \\
\hline
\end{tabular}

\begin{tabular}{l|l|}
\hline$y=0.2351 x+0.7382$ & $R^{2}=0.6513$ \\
\hline$y=0.7389 x+3.7263$ & $R^{2}=0.9995$ \\
\hline
\end{tabular}

\begin{tabular}{ll}
\hline$y=0.7389 x+3.7263$ & $R^{2}=0.9995$ \\
\hline$y=0.022 x+13.556$ & $R^{2}=0.9542$ \\
\hline
\end{tabular}

\begin{tabular}{|l|l|}
\hline$y=0.022 x+13.556$ & $R^{2}=0.9542$ \\
\hline
\end{tabular}

\begin{tabular}{|l|l|}
\hline$y=0.8116 x+4.106$ & $R^{2}=0.9497$ \\
\hline$y=0.511 x+1.6487$ & $R^{2}=0.928$ \\
\hline
\end{tabular}

\begin{tabular}{|l|l|}
\hline$y=0.8116 \mathrm{x}+4.106$ & $\mathrm{R}^{2}=0.9497$ \\
\hline $\mathrm{y}=0.4511 \mathrm{x}+1.6487$ & $\mathrm{R}^{2}=0.928$ \\
\hline
\end{tabular}

\begin{tabular}{|l|l|}
\hline$y=0.5228 x+1.8536$ & $R^{2}=0.8698$ \\
\hline$y=0.178 x+5.474$ & $R^{2}=0.5821$ \\
\hline
\end{tabular}

\begin{tabular}{|l|l|}
\hline$y=0.528 x+1.8536$ & $R^{2}=0.8698$ \\
\hline$y=0.178 x+5.4714$ & $R^{2}=0.5821$ \\
\hline$y=0.3209 x+1.1013$ & $R^{2}=0.9469$ \\
\hline
\end{tabular}

\begin{tabular}{|l|l|}
\hline$y=0.3209 x+1.1013$ & $R^{2}=0.9469$ \\
\hline$y$
\end{tabular}

\begin{tabular}{|l|l|}
\hline$y=0.2607 x+6.2904$ & $R^{2}=0.7629$ \\
\hline
\end{tabular}

\begin{tabular}{|l|l|}
\hline $\mathrm{y}=0.2284 \mathrm{x}+3.5747$ & $\mathrm{R}^{2}=0.8731$ \\
\hline
\end{tabular}

\begin{tabular}{|l|l|}
\hline$y=0.2813 x+3.7933$ & $R^{2}=0.6183$ \\
\hline$y=0.6596 x+2.1542$ & $R^{2}=0.9437$ \\
\hline
\end{tabular}

\begin{tabular}{|l|l|}
\hline $\mathrm{y}=0.28596 \mathrm{x}+2.1542$ & $\mathrm{R}^{2}=0.9437$ \\
\hline $\mathrm{y}=0.242 \mathrm{x}+4.9552$ & $\mathrm{R}^{2}=0.976$ \\
\hline
\end{tabular}

\begin{tabular}{|l|l|}
\hline$y=0.2429 x+4.9552$ & $R^{2}=0.9976$ \\
\hline$y=0.6142 x+2.525$ & $R^{2}=0.7772$ \\
\hline
\end{tabular}

\begin{tabular}{|l|l|}
\hline$y=0.6142 x+2.525$ & $R^{2}=0.7772$ \\
\hline
\end{tabular}

\begin{tabular}{|l|l|}
\hline$y=0.1283 x+13.168$ & $R^{2}=0.9694$ \\
\hline$y=0.1768 x+5.639$ & $R^{2}=0.886$ \\
\hline
\end{tabular}

\begin{tabular}{|l|l|}
\hline$y=0.1768 x+5.6639$ & $R^{2}=0.8816$ \\
\hline$y=0.5096 x+0.939$ & $R^{2}=0.9167$ \\
\hline
\end{tabular}

\begin{tabular}{|l|l|}
\hline$y=0.5096 x+0.939$ & $R^{2}=0.9167$ \\
\hline$y=0.244 x-2.4476$ & $R^{2}=0.999$ \\
\hline
\end{tabular}

\begin{tabular}{ll}
$y=0.4244 x-2.4476$ & $R^{2}=0.9999$ \\
\hline$y=0.136 x+18.064$ & $R^{2}=0.9844$ \\
\hline
\end{tabular}

\begin{tabular}{ll}
$y=0.1368 x+18.064$ & $R^{2}=0.9844$ \\
\hline$y=0.493 x+4.5651$ & $R^{2}=0.996$ \\
\hline
\end{tabular}

\begin{tabular}{|l|l|}
\hline$y=0.493 x+4.5651$ & $R^{2}=0.996$ \\
\hline$y=0.3472 x+3.5028$ & $R^{2}=0.9668$ \\
\hline
\end{tabular}

\begin{tabular}{ll}
$y=0.3472 x+3.5028$ & $R^{2}=0.9668$ \\
\hline$y=0.213 x+6.8758$ & $R^{2}=0.822$ \\
\hline
\end{tabular}

\begin{tabular}{ll}
\hline $\mathrm{y}=0.2133 \mathrm{x}+6.8758$ & $\mathrm{R}^{2}=0.822$ \\
\hline $\mathrm{y}=0.7757 \mathrm{x}-8.777$ & $\mathrm{R}^{2}=0.9454$
\end{tabular}

\begin{tabular}{|l|l|}
\hline$y=0.213 x+6.8758$ & $R^{2}=0.822$ \\
\hline$y=0.7757 x-8.777$ & $R^{2}=0.9454$ \\
\hline
\end{tabular}

\begin{tabular}{|l|l|}
\hline$y=0.3243 x+2.8201$ & $R^{2}=0.7689$ \\
\hline$y=0.3001 x+8.5566$ & $R^{2}=0.9999$ \\
\hline
\end{tabular}

\begin{tabular}{ll|l}
\hline $\mathrm{y}=0.3001 \mathrm{x}+8.5566$ & $\mathrm{R}^{2}=0.9999$ \\
\hline $\mathrm{y}=0.3248 \mathrm{x}+2.1631$ & $\mathrm{R}^{2}=1$ \\
\hline
\end{tabular}

\begin{tabular}{|l|l|}
\hline$y=0.3248 x+2.1631$ & $R^{2}=1$ \\
\hline
\end{tabular}

\begin{tabular}{|l|l|}
\hline$y=0.0555 x+9.3178$ & $R^{2}=0.999$ \\
\hline$y=0.2659 x+14.085$ & $R^{2}=0.8876$ \\
\hline
\end{tabular}

\begin{tabular}{|l|l|}
\hline$y=0.2659 x+14.085$ & $R^{2}=0.8876$ \\
\hline$y=0.455 x+0.587$ & $R^{2}=0.958$ \\
\hline
\end{tabular}

\begin{tabular}{|l|l|}
\hline$y=0.4455 x+0.0587$ & $R^{2}=0.9518$ \\
\hline
\end{tabular}

Linear fitting $\mathrm{R}$ square

equation

Fig. 7 The expression of genes and regulators related to carotenoid metabolism in the visualized networks for the 'blue' and 'turquoise' modules. a Heatmap of the expression of structural genes related to carotenoid biosynthesis during fruit ripening. Columns and rows in the heat map represent the developmental stage and gene name, respectively. The black and blue genes represent structural genes and transcription factors, respectively. Red, green and white indicate high expression, low expression and the absence (or undetectable levels) of detectable transcripts at the corresponding stage, respectively. $\mathbf{b}$ The linear fitting equation and R squared values between the FPKM and qRT-PCR values

in a decrease in carotenoid content [30]. In this study, we observed that the expression level of HY5 gradually decreased during ripening, in parallel with the decline in carotenoid levels in apricot fruit. In Arabidopsis, HY5 and PIFs form a dynamic activation-suppression transcriptional module that is responsive to light and temperature cues and regulates carotenoid and chlorophyll accumulation by direct binding to the same G-box cis element of a target gene [31]. However, more work is required to confirm whether this fine-tuning module exists in fruit. Taken together, these results suggest that light signalingassociated transcription factors are involved in regulating carotenoid accumulation in ripening apricot fruit.

Previous studies have shown that the synthesis and accumulation of carotenoids are regulated by various phytohormones. Tomato fruit ripening is triggered by an 


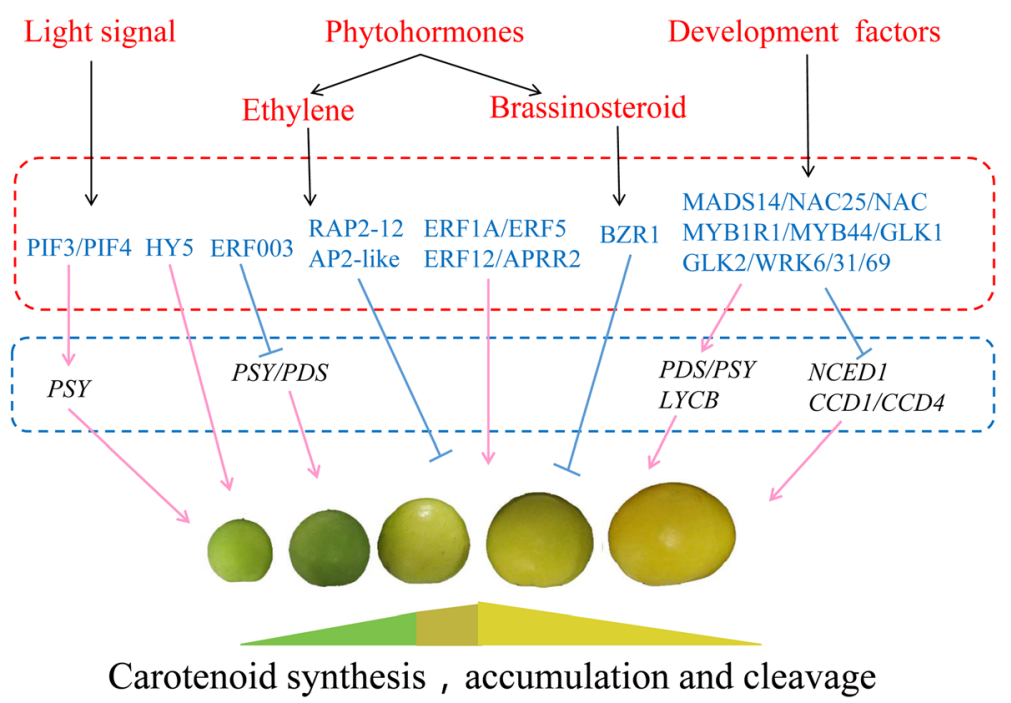

Fig. 8 The proposed model of carotenoid metabolism in apricot during fruit ripening. Pink arrows represent positive regulation; blue lines represent negative regulation; black and skyblue genes represent structural genes and transcription factors, respectively. PIF, phytochrome interacting factor; $\mathrm{HY} 5$, LONG HYPOCOTYL5; RAP2-12, Ethylene-responsive transcription factor RAP2-12; ERF, Ethylene response factor; BZR1, BRASSINAZOLE RESISTANT1; MADS14, MADS-box transcription factor 14; NAC, NAC transcription factor; MYB, MYB transcription factor; GLK, GOLDEN 2-LIKE transcription factor

increase in ethylene biosynthesis and is accompanied by a large increase in $\beta$-carotene and lycopene content as well as the expression of SIPSY1 and SIPDS, which is ethylene dependent [32]. Similarly, ethylene induces the expression of PSY1 and PDS and promotes the accumulation of the corresponding carotenoid product in apricot [32]. This is consistent with our observation that the upregulation of the expression of PDS1 (CL1571.2) and the ethylene response factor ERFO03 was correlated with increased phytoene and neoxanthin levels during ripening. We propose that PDS1 may also be regulated by ethylene in apricots to promote carotenoid accumulation (Fig. 1c and Fig. 7). In tomato, the suppression of SIAP $2 a$ mediated by RNAi results in lower levels of carotenoids, and reduced expression of SIERF6 enhances carotenoid accumulation, suggesting that these genes play positive and negative roles, respectively, in carotenoid accumulation during ripening [33, 34]. In our study, ERF genes showed two distinct expression patterns, indicating that ethylene signaling member has different functions in the regulation of carotenoid accumulation in apricot fruit (Fig. 7a). In A. thaliana, the ERF gene RAP2.2 directly binds to the AtPSY and AtPDS promoters to reduce carotenoid accumulation [12]. Here, we also observed that the increased expression levels of RAP2-12 showed a negative correlation with the levels of carotenoid accumulation, while ERF1A, ERF5/12, and $A P R R 2$ were positively correlated with carotenoid accumulation (Fig. 7a).

In other studies, the application of the brassinosteroid (BR) 2,4-epibrassinolide (EBR) to tomato has led to increased levels of lycopene and a reduced chlorophyll content in the fruit [35]. Additionally, the transcription factor BRASSINAZOLE RESISTANT1 (BZR1) has been shown to play a role in carotenoid accumulation in tomato fruit [36]. In this study, we observed increased expression of $B Z R 1$ during ripening, suggesting that the $B R$ signaling pathway may also be associated with carotenoid accumulation (Fig. 7b). Over time, our results are consistent with the regulation of carotenoid accumulation during apricot fruit ripening by both ethylene and BR.

Numerous developmental regulatory factors have been shown to affect the synthesis and accumulation of carotenoids. These include the MADS-box genes AGAMOUSLIKE 1 and FRUITFUL1, which modulate carotenoid accumulation during tomato fruit ripening [37], and the MADS-box transcription factors RIN and FUL1/TDR4, which directly bind to the SIPSY1 and SIPDS1 promoters to regulate carotenoid synthesis $[38,39]$. Recently, a sweet orange (Citrus sinensis) transcription factor, CsMADS6, was found to promote carotenoid accumulation by coordinately modulating the expression of $L C Y B 1, P S Y, P D S$ and $C C D 1$ [40]. Here, we observed that the downregulation of MADS14 expression was accompanied by upregulated expression of $C C D 1 / 4$ and downregulated expression of PDS1 (U17285), consistent with their involvement in carotenoid accumulation during ripening (Fig. 1c and Fig. 7a). NAC transcription factors are plant specific and play important roles in the development of various organs and fruit [41]. Overexpression of SINAC1 and SINAC4 results in reduced carotenoid levels and inhibition of fruit ripening by altering the carotenoid pathway flux and decreasing the levels of ethylene biosynthesis genes belonging to system-2, but a newly identified tomato NAC (N AM/A TAF1/2/C 
UC2), SINAC4, functions as a positive regulator of fruit ripening and carotenoid accumulation [41, 42]. In this study, we observed that three NAC (NAC25, NAC U12188, NAC_CL3330.2) genes with different expression patterns were involved in regulating carotenoid accumulation (Fig. 7a).

MYB TFs regulate diverse plant developmental processes. The R2R3-MYB TF RCP1 is a master regulator that controls the entire CBP during flower development and positively regulates carotenoid biosynthesis during Mimulus lewisii flower development [43]. MYB7 can activate the $A d L C Y B$ promoter, thereby modulating carotenoid pigment accumulation in kiwifruit (Actinidia deliciosa) [44]. Similarly, the significantly reduced expression of MYB1R1 and MYB44 during ripening suggested their involvement in carotenoid accumulation in apricots (Fig. 7a). In contrast, CrMYB68 suppresses the expression of $\mathrm{CrBCH} 2$ and $\mathrm{CrNCED} 5$ in the citrus carotenoid pathway [45]. Another MYB transcription factor, GOLDEN2-LIKE (GLK2), has been shown to increase lycopene levels in tomato fruit at the red ripe stage [46-48]. Here, the expression levels of GLK1 and GLK2 decreased during ripening, suggesting that their expression may limit carotenoid biosynthesis during ripening in apricots (Fig. 7a). Overexpression of SIPRE2, an atypical bHLH transcription factor, negatively affects plant morphology and fruit pigment accumulation in tomato by downregulating the expression of the chlorophyll-related genes GLK2 and RbcS and the carotenoid biosynthesis-related genes PSY1A and ZDS in ripening tomato [49], suggesting that bHLH TFs may regulate carotenoids upstream of GLKs. In the present study, we identified three bHLHs with downregulated expression patterns, but their regulatory roles are still unknown. We also examined the expression patterns of the WRKY6/31/69 transcription factors (Fig. 7b), whose homolog OfWRKY3 has been implicated as a positive regulator of the OfCCD4 gene in Osmanthus fragrans [50]. Collectively, these results indicated that many developmental factors take part in regulating carotenoid biosynthesis and accumulation in apricots.

We noted that higher expression levels of PDS1, ZEP and VDE1 were detected in 'LT' apricot fruit. In addition, the two genes responsible for carotenoid cleavage (CCD1 and $C C D 4)$ exhibited remarkable upregulated expression patterns in the 'carotenoid loss' type of cultivar during fruit ripening (Fig. 7a). Similarly, studies in strawberry showed that changes in lutein content were closely related to the expression level of $C C D 1$ during ripening [51]. CCD4 has also been shown to control peach flesh pigmentation, with high $C C D 4$ transcript abundance being observed in white flesh peach, which is associated with the emission of carotenoid-derived volatiles [52]. In this study, the upregulated expression of two ZEP genes (U10800 and U21300) was accompanied by a decrease in violaxanthin during ripening. Loss of function of $Z E P$ in the aba1 mutant of $A$. thaliana and the $a b a 2$ mutant of tobacco causes the accumulation of high zeaxanthin levels in leaves [53, 54]. These observations suggest that $Z E P, C C D 1$ and $C C D 4$ play central roles in determining carotenoid levels in apricots and that degradation is the key control point for carotenoid accumulation.

\section{Conclusions}

In this study, WGCNA analysis revealed that two modules ('blue' and 'turquoise') are highly correlated with carotenoid metabolism. Structural genes (CCD1, CCD4, VDE1, ZEP and $P D S 1)$ are associated with the carotenoid biosynthesis pathway, and transcription factors related to light signaling (PIF3/4 and HY5), phytohormones (ERF4/5/12, AP2, AP2like and BZR1) and development factors (MADS14, NAC2/ 25, MYB1R1/44, GLK1/2, and WRKY6/31/69) may play important regulatory roles in carotenoid metabolism, but the specific regulatory mechanism still needs to be elucidated. Our findings not only provide new insights into the mechanisms of carotenoid metabolism, but these datasets also provide a useful platform for further functional studies of candidate genes.

\section{Methods}

\section{Plant materials}

From May to July 2017, fruits from the 'LT' cultivar were harvested at different ripening stages (turning, T, 57 DPA (days post anthesis); commercial maturation, CM, 65 DPA and fully ripe, FR, 74 DPA) from the National Fruit Tree Germplasm Repository, Academy of Xinjiang Agricultural Sciences, Luntai, Xinjiang, China (Fig. 1a). All samples were transported to the laboratory on the day of harvest. A set of fruit of uniform size and with no obvious mechanical damage was selected for the subsequent experiments. Fifty fruits were used as one replicate, and three biological replicates were used for each sample. For each replicate, 20 fruit were used to measure a range of physiological indices. Other fruit were cut into small cubes and then immediately frozen in liquid nitrogen and stored at $-80^{\circ} \mathrm{C}$ for measurement of carotenoid contents and for RNA-Seq and gene expression analysis.

\section{Determination of basic quality parameters}

Flesh firmness was measured using a firmness tester (Model: HL-300, Xianlin Non Detection Device Co., Ltd., Nanjing, China) with an $8 \mathrm{~mm}$ probe. To determine TSS values, the juice was squeezed from three fruits per replicate, pooled and analyzed using a handheld digital refractometer (B32T Brix Meter, Guangzhou Ruiqi Trading Company, Guangdong, China). TA values were determined after the juice sample was diluted 100 times with pure water. The fruit color parameters were measured using a Hunter's Mini Scanning Colorimeter (Hunter Associates Laboratory, Inc., Reston, VA, USA). 
The CCI was calculated with the following formula: $\mathrm{CCI}=1000 \times \mathrm{a}^{*} /\left(\mathrm{L}^{*} \times \mathrm{b}^{*}\right)$, using three fruits as a single replicate and three biological replicates.

\section{Extraction and identification of carotenoids}

Carotenoids were extracted as previously described with some modifications [55]. Eight grams of fruit peel was placed in a screw-top tube, and $50 \mathrm{~mL}$ of extraction solvent (hexane/acetone/ethanol, 50:25:25, v/v) was added. After standing for $30 \mathrm{~min}$, the samples were centrifuged for $5 \mathrm{~min}$ at $6500 \mathrm{rpm}$. The colored top layer of hexane was recovered and transferred to a volumetric flask, where it was dried under nitrogen, and dissolved in $2 \mathrm{~mL}$ of methyl tert-butyl ether (MTBE) before being combined with $2 \mathrm{~mL}$ of $10 \%$ methanol/potassium hydroxide. The mixture was allowed to stand for $1 \mathrm{~h}$ and separated in a separating funnel and was rinsed twice with water and once with $0.1 \%$ butylhydroxytoluene (BHT)/MTBE. The rinsed solution was transferred to a brown bottle and dried under nitrogen before 2 $\mathrm{mL}$ methanol/acetone $(2,1)$ was added, and the solution was filtered through a $0.22 \mathrm{~m}$ filter membrane.

Carotenoids were identified by high-pressure liquid chromatography (HPLC, Waters, Milford, MA, USA) with a C30 chromatography column $(250 \mathrm{~mm} \times 4.6 \mathrm{~mm}, 5 \mu \mathrm{m}$, YMC, Wilmington, NC, USA). The mobile phase flow rate was $1 \mathrm{~mL} / \mathrm{min}$, and the column temperature was $25^{\circ} \mathrm{C}$, with a detection wavelength of $450 \mathrm{~nm}$ and an injection volume of $20 \mu \mathrm{L}$. The mobile phase composition was as follows: methanol, MTBE and water. Carotenoids were identified by comparison to standard retention times and UV-visible spectral peaks. The quantification of carotenoids was performed using a standard curve and expressed as $\mu \mathrm{g} / \mathrm{g}$ FW. Three biological replicates for each sample were used.

\section{RNA extraction, library construction and data analysis}

Total RNA was isolated and extracted from $1 \mathrm{~g}$ of fruit peel for each sample using a Tiangen reagent kit (Tiangen, Beijing, China). RNA purity, concentration and integrity were determined using a Nanodrop 2000 (NanoDrop 2000, Wilmington, DC, USA) and denaturing agarose gel electrophoresis. 'LT' RNA-Seq libraries of T-, CM- and FR-stage fruit were constructed using an Illumina TruSeq RNA Library Prep Kit v2 following standard procedures, and three biological replicates were used for each stage. The libraries were sequenced using an Illumina HiSeq ${ }^{\text {Tm }} 2000$ system at the Beijing Genomics Institute (BGI), China. Clean reads were obtained by removing the linker, repetitive, redundant and low-quality sequences from the raw reads. The sequence data from this study have been deposited in the National Center for Biotechnology Information (NCBI) under Sequence Read Archive (SRA) accession number PRJNA530709.

\section{De novo assembly and functional annotation}

De novo transcriptome assembly was carried out using the short read assembly program Trinity (http://trinityrnaseq.sourceforge.net/) [56]. The assembled unigenes were first filtered to remove redundant sequences and further spliced using the TGICL (https://sourceforge. net/projects/tgicl/files/tgicl\%20v2.1/) version 2.1 software package [57]. The sequences were then clustered into homologous transcripts to obtain the final unigenes. Subsequently, the unigenes were divided into two classes: one with several unigenes with $>70 \%$ similarity, with the prefix CL followed by the cluster ID, and the other consisting of singletons, with the prefix unigene. Finally, BLASTx alignment (e-value $<0.00001$ ) was performed between the unigenes and the NR, Swiss-Prot, KEGG and COG protein databases, and the best alignments were used to determine the sequence direction of the unigenes. The GO annotations of unigenes were obtained using Blast2GO Version 2.3.4 software (https:// www.blast2go.com) [58]. WEGO Version 2.0 (Web Gene Ontology Annotation Plot) software (http://wego.genomics.org.cn) was used to provide the GO functional classifications of all the unigenes [59].

Principal component analysis (PCA) and identification of DEGs PCA was performed to verify the reproducibility of the sequence data using a previously described method [60]. DEGs between each sample were identified on the basis of FPKM values [61] and pairwise comparisons, with a false discovery rate (FDR) threshold of $<0.001$ and an absolute value of the $\log 2$ Ratio $\geq 1$ using the DESeq $R$ package (v1.10.1) [62]. The DEGs were also analyzed by heatmap clustering using Origin Pro 2018 (Origin Lab, Northampton, MA, USA).

\section{GO and KEGG pathway enrichment analysis}

The DEGs were mapped to each term in the GO database (http://www.geneontology.org/) [63], and the number of genes associated with each term was determined. Based on the gene numbers for each GO term, a hypergeometric test was applied to determine the GO terms that were significantly enriched for DEGs compared with the genome background. A corrected $p$-value $<0.05$ was used as the threshold, and the GO terms meeting this condition were defined as significantly enriched for DEGs. A KEGG pathway enrichment analysis was performed using the KEGG database (http://www.genome. jp/kegg/) [64]. After multiple tests and corrections, we determined that pathways with a Q-value 0.05 were significantly enriched in DEGs.

\section{WGCNA and visualization of gene networks}

A total of 16,773 unigenes with FPKM values $>1$ were used to perform WGCNA by using the WGCNA 
package in $\mathrm{R}$ [26]. The modules were obtained using the automatic network construction function. Modules were identified using default settings, except that the soft power was 10 , the min module size was 30 , and the merge cut height was 0.25 . Finally, Cytoscape version 2.8 (http://www.cytoscape.org/) was used to visualize the carotenoid metabolism regulatory network based on the WGCNA modules [65].

\section{Real-time quantitative PCR}

The expression levels of candidate structural genes and transcription factors were measured in 'LT' fruit at three ripening stages by qRT-PCR analysis. Ribosomal RNA and actin gene expression was used as a normalization reference as previously described [24]. Specific primers were designed using Primer5 (Additional file 9). Gene expression levels were detected using an iQ5 instrument (Bio-Rad Laboratories, Inc. America) with the SYBR ${ }^{\circ}$ Premix Ex TaqTM II Kit (TaKaRa Biotechnology (Dalian) Co, Ltd., China). The amplification program was as follows: $95^{\circ} \mathrm{C}$ for $1 \mathrm{~min}$, followed by 40 cycles at $95^{\circ} \mathrm{C}$ for $20 \mathrm{~s}, 58^{\circ} \mathrm{C}$ for $20 \mathrm{~s}$ and $72{ }^{\circ} \mathrm{C}$ for $30 \mathrm{~s}$. Each qRT-PCR analysis was performed in triplicate, and the mean value was used for the qRT-PCR analysis. The relative expression of the genes was calculated according to the 2 $-\triangle \Delta C T$ method [66], and OriginPro 2018 (OriginLab, Northampton, MA, USA) was used to analyze the data.

\section{Supplementary information}

Supplementary information accompanies this paper at https://doi.org/10. 1186/s12864-019-6261-5.

Additional file 1. Throughput and quality of RNA-Seq data in apricot fruit. Additional file 2. Statistics of assembly quality.

Additional file 3. Gene ontology (GO) classification map. The horizontal axis represents the type of $\mathrm{GO}$ function; the vertical axis on the right represents the number of unigenes annotated to the corresponding $\mathrm{GO}$ function; and the vertical axis on the left represents the percentage of the number of unigenes within the total.

Additional file 4. Differentially expressed genes (DEGs) shown in Fig. 3b. Additional file 5. Gene ontology (GO) annotation of differentially expressed genes (DEGs). (A) T vs CM, (B) T vs FR, (C) CM vs FR. The unigenes were annotated in three categories: biological process, cellular component and molecular functions. The right $y$-axis indicates the number of genes in a category, and the left $y$-axis indicates the percentage of a specific gene.

Additional file 6. Significantly enriched differentially expressed genes (DEGs) in Kyoto Encyclopedia of Genes and Genomes (KEGG) pathways during different ripening stages.

Additional file 7. The expression and functional annotation of genes shown in Fig. 5b.

Additional file 8. The expression and functional annotation of genes shown in Fig. 6.

Additional file 9. Primer sequences used for real-time quantitative PCR.

\section{Abbreviations}

AP2: Ethylene-responsive transcription factor AP2; bHLH: Basic helix-loophelix protein; BHT: Butylhydroxytoluene; BR: Brassinosteroid;
BZR1: BRASSINAZOLE RESISTANT1; CCD1/4: Carotenoid cleavage dioxygenase 1/4; CCl: Citrus color index; CCS: Capsanthin/capsorubin synthase; CHYB: $\beta$ carotene hydroxylase; CM: Commercial maturation; COP1-like: CONSTITUTIVE PHOTOMORPHOGENIC 1-like; CRTISO: Carotenoid isomerase;

DEGs: Differentially expressed genes; DMAPP: Dimethylallyl diphosphate; DXS: Deoxy-d-xylulose-5-phosphate synthase; EBR: 2,4-epibrassinolide; ERF003/5/12: Ethylene-responsive transcription factor 003/5/12; FDR: False discovery rate; FPKM: Fragments per kilobase of transcript per million mapped reads; FR: Fully ripe; FW: Fresh weight; GGPP: Geranylgeranyl diphosphate; GGPPS: Geranylgeranyl diphosphate synthase; GLK1/2: GOLDEN 2-LIKE transcription factor; GO: Gene ontology; HPLC: High-pressure liquid chromatography; IPP: Isopentenyl diphosphate isomerase; KEGG: Kyoto Encyclopedia of Genes and Genomes; LCYB: $\beta$-cyclase; LCYE: $\varepsilon$-cyclase; LT: Luntaixiaobaixing; MADS14: MADS-box transcription factor 14; MEP: Methyl-D-erythritol 4-phosphate; MTBE: Methyl tert-butyl ether; MYB1R1/44: Transcription factor MYB1R1/44; MYC2: Transcription factor MYC2; NAC2/25: Transcription factor NAC2/25; NCED1: 9-cis-epoxycarotenoid dioxygenase 1; NXS: Neoxanthin synthase; PCA: Principal component analysis; PDS1: Phytoene desaturase 1; PIF3/4: Phytochrome interacting factor 3/4; PSY: Phytoene synthase; qRT-PCR: Quantitative real time reverse transcriptase PCR; RAP2-12: Ethylene-responsive transcription factor RAP2-12; RIN: Ripening inhibitor; RNA-Seq: RNA sequencing; SRA: Sequence Read Archive; T: Turning; TA: Titratable acidity; TSS: Total soluble solids; VDE1: Violaxanthinde-epoxidase 1; WGCNA: Weighted gene coexpression network analysis; WRKY6/31/69: Transcription factor WRKY6/31/69; ZDS: そcarotene desaturase; ZEP: Zeaxanthin epoxidase; Z-ISO: 15-cis-Zeta-carotene isomerase.

\section{Acknowledgements}

We thank Junhui Zhou (Department of Cell Biology and Molecular Genetics, University of Maryland) for comments on an earlier version of this manuscript.

\section{Authors' contributions}

WX designed the project. LZ measured the carotenoids and prepared the manuscript. QZ analyzed the data. WL participated in collecting the materials. SZ participated in assaying the physiological parameters. All authors read and approved the final manuscript.

\section{Funding}

This work was supported by the National Natural Science Foundation of China (No. 31872046). The funding body played no role in the design of the study and collection, analysis, and interpretation of data and in writing the manuscript.

\section{Availability of data and materials}

The datasets supporting the results of this article are included within the article and its additional files. All of the obtained sequences from apricot were deposited in the NCBI Sequence Read Archive (SRA) repository under accession number PRJNA530709 and were released on April 3, 2019.

Ethics approval and consent to participate

Not applicable.

Consent for publication

Not applicable.

\section{Competing interests}

The authors declare that they have no competing interests.

\section{Author details}

${ }^{1}$ College of Horticulture and Landscape Architecture, Southwest University, Chongqing 400716, People's Republic of China. '2Laboratory of Fruit Quality Biology, Zhejiang University, Zijingang Campus, Hangzhou 310058, People's Republic of China. ${ }^{3}$ Agriculture National Fruit Tree Germplasm Repository, Xinjiang Academy of Agricultural Sciences, Luntai, Xinjiang 841600, People's Republic of China. 


\section{Received: 9 August 2019 Accepted: 5 November 2019} Published online: 20 November 2019

\section{References}

1. Yuan H, Zhang J, Nageswaran D, Li L. Carotenoid metabolism and regulation in horticultural crops. Hortic Res. 2015;2:15036.

2. Nakabayashi R, Saito K. Integrated metabolomics for abiotic stress responses in plants. Curr Opin Plant Biol. 2015;24:10-6.

3. Rao AV, Rao LG. Carotenoids and human health. Pharmacol Res. 2007;55(3): 207-16.

4. Nisar N, Li L, Lu S, Khin NC, Pogson BJ. Carotenoid metabolism in plants. Mol Plant. 2015;8(1):68-82.

5. Sun T, Yuan H, Cao H, Yazdani M, Tadmor Y, Li L. Carotenoid metabolism in plants: the role of plastids. Mol Plant. 2018;11(1):58-74.

6. Giuliano G, Aquilani R, Dharmapuri S. Metabolic engineering of plant carotenoids. Trends Plant Sci. 2000;5(10):406-9.

7. Hirschberg J. Carotenoid biosynthesis in flowering plants. Curr Opin Plant Biol. 2001;4(3):210-8

8. Tal I, Gil R, Dani Z, Joseph $\mathrm{H}$. Cloning of tangerine from tomato reveals a carotenoid isomerase essential for the production of beta-carotene and xanthophylls in plants. Plant Cell. 2002;14(2):333-42.

9. Ronen G, Cohen M, Zamir D, Hirschberg J. Regulation of carotenoid biosynthesis during tomato fruit development: expression of the gene for lycopene epsilon-cyclase is down-regulated during ripening and is elevated in the mutant Delta. Plant J. 1999;17(4):341-51.

10. Fraser PD, Truesdale MR, Bird CR, Schuch W, Bramley PM. Carotenoid biosynthesis during tomato fruit development (evidence for tissue-specific gene expression). Plant Physiol. 1994;105(1):405-13.

11. Lois LM, Rodríguez-Concepción M, Gallego F, Campos N, Boronat A. Carotenoid biosynthesis during tomato fruit development: regulatory role of 1-deoxy-D-xylulose 5-phosphate synthase. Plant J. 2000;22(6):503-13.

12. Welsch R, Maass D, Voegel T, DellaPenna D, Beyer P. Transcription factor RAP2. 2 and its interacting partner SINAT2: stable elements in the carotenogenesis of Arabidopsis leaves. Plant Physiol. 2007;145(3):1073-85.

13. Toledo-Ortiz G, Huq E, Rodríguez-Concepción M. Direct regulation of phytoene synthase gene expression and carotenoid biosynthesis by phytochrome-interacting factors. Proc Natl Acad Sci U S A. 2010;107(25): 11626-31.

14. Catherine M, Julia V, Petra T, Giovannoni JJ. The tomato MADS-box transcription factor RIPENING INHIBITOR interacts with promoters involved in numerous ripening processes in a COLORLESS NONRIPENING-dependent manner. Plant Physiol. 2011;157(3):1568-79.

15. Ruizsola MÁ, Rodríguezconcepción M. Carotenoid biosynthesis in Arabidopsis: a colorful pathway. In: Bak S, Beisson F, Bishop G, Hamberger B, Höfer R, Paquette S, Werck-Reichhart D, editors. The Arabidopsis book. Rockville: MD American Society of Plant Biologists; 2012. p. 1-28.

16. Fratianni F, Ombra MN, d'Acierno A, Cipriano L, Nazzaro F. Apricots: biochemistry and functional properties. Curr Opin Food Sci. 2018;19:23-9.

17. Xi W, Zheng H, Zhang Q, Li W. Profiling taste and aroma compound metabolism during apricot fruit development and ripening. Int J Mol Sci. 2016;17:998

18. Dillies MA, Rau A, Aubert J, Hennequet-Antier C, Jeanmougin M, Servant N, et al. A comprehensive evaluation of normalization methods for Illumina high-throughput RNA sequencing data analysis. Brief Bioinform. 2013;14(6): 671-83.

19. Malone $\mathrm{JH}$, Oliver B. Microarrays, deep sequencing and the true measure of the transcriptome. BMC Biol. 2011;9:34.

20. Li G, Ma J, Tan M, Mao J, An N, Sha G, et al. Transcriptome analysis reveals the effects of sugar metabolism and auxin and cytokinin signaling pathways on root growth and development of grafted apple. BMC Genomics. 2016; 17(1):150.

21. Garcia-Seco D, Zhang Y, Gutierrez-Mañero FJ, Martin C, Ramos-Solano B. RNA-Seq analysis and transcriptome assembly for blackberry (Rubus sp. Var. Lochness) fruit. BMC Genomics. 2015;16(1):5

22. Yu K, Xu Q, Da X, Guo F, Ding Y, Deng X. Transcriptome changes during fruit development and ripening of sweet orange (Citrus sinensis). BMC Genomics. 2012;13(1):10.

23. Sánchez-Sevilla JF, Cruz-Rus E, Valpuesta V, Botella MA, Amaya I. Deciphering gamma-decalactone biosynthesis in strawberry fruit using a combination of genetic mapping, RNA-Seq and eQTL analyses. BMC Genomics. 2014;15(1):218.
24. Zhang Q, Feng C, Li W, Qu Z, Zeng M, Xi W. Transcriptional regulatory networks controlling taste and aroma quality of apricot (Prunus armeniaca L. ) fruit during ripening. BMC Genomics. 2019;20(1):45.

25. Manganaris GA, Rasori A, Bassi D, Geuna F, Ramina A, Tonutti P, et al. Comparative transcript profiling of apricot (Prunus armeniaca L.) fruit development and on-tree ripening. Tree Genet Genomes. 2011;7(3):609-16.

26. Langfelder $P$, Horvath S. WGCNA: an R package for weighted correlation network analysis. BMC Bioinformatics. 2008;9:559.

27. Llorente B, Martinez-Garcia JF, Stange C, Rodriguez-Concepcion M. Illuminating colors: regulation of carotenoid biosynthesis and accumulation by light. Curr Opin Plant Biol. 2017;37:49-55.

28. Leivar P, Monte E. PIFs: systems integrators in plant development. Plant Cell. 2014;26(1):56-78.

29. Mannen K, Matsumoto T, Takahashi S, Yamaguchi Y, Tsukagoshi M, Sano R, et al. Coordinated transcriptional regulation of isopentenyl diphosphate biosynthetic pathway enzymes in plastids by phytochrome-interacting factor 5. Biochem Biophys Res Commun. 2014;443(2):768-74.

30. Yongsheng L, Sherry R, Zhibiao Y, Cornelius B, Ageeth VT, Julia V, et al. Manipulation of light signal transduction as a means of modifying fruit nutritional quality in tomato. Proc Natl Acad Sci U S A. 2004;101(26):9897902.

31. Toledo-Ortiz G, Johansson H, Lee KP, Bou-Torrent J, Stewart K, Steel G, et al. The HY5-PIF regulatory module coordinates light and temperature control of photosynthetic gene transcription. PLoS Genet. 2014;10(6):e1004416.

32. Marty I, Bureau S, Sarkissian G, Gouble B, Audergon JM, Albagnac G. Ethylene regulation of carotenoid accumulation and carotenogenic gene expression in colour-contrasted apricot varieties (Prunus armeniaca). J Exp Bot. 2005;56(417):1877-86.

33. Chung M-Y, Vrebalov J, Alba R, Lee J, McQuinn R, Chung J-D, et al. A tomato (Solanum lycopersicum) APETALA2/ERF gene, SIAP2a, is a negative regulator of fruit ripening. Plant J. 2010;64(6):936-47.

34. Lee JM, Joung JG, Mcquinn R, Chung MY, Fei Z, Tieman D, et al. Combined transcriptome, genetic diversity and metabolite profiling in tomato fruit reveals that the ethylene response factor SIERF6 plays an important role in ripening and carotenoid accumulation. Plant J. 2012;70(2):191-204.

35. Vardhini B. Vidya, Rao SSR. Acceleration of ripening of tomato pericarp discs by brassinosteroids. Phytochemistry. 2002;61(7):843-7.

36. Liu L, Jia C, Zhang M, Chen D, Chen S, Guo R, et al. Ectopic expression of a BZR1-1D transcription factor in brassinosteroid signalling enhances carotenoid accumulation and fruit quality attributes in tomato. Plant Biotechnol. 2014;12(1):105-15.

37. Julia V, Pan IL, Arroyo AJM, Ryan MQ, Miyoung C, Mervin P, et al. Fleshy fruit expansion and ripening are regulated by the tomato SHATTERPROOF gene TAGL1. Plant Cell. 2009;21(10):3041-62.

38. Masaki F, Toshitsugu N, Yoko S, Yasuhiro I. A large-scale identification of direct targets of the tomato MADS box transcription factor RIPENING INHIBITOR reveals the regulation of fruit ripening. Plant Cell. 2013;25(2):371-86.

39. Masaki F, Yoko S, Hiroyuki N, Mamiko K, Junji K, Toshitsugu N, et al. Transcriptional regulation of fruit ripening by tomato FRUITFULL homologs and associated MADS box proteins. Plant Cell. 2014;26(1):89-101.

40. Lu S, Zhang Y, Zhu K, Yang W, Ye J, Chai L, et al. The citrus transcription factor CSMADS6 modulates carotenoid metabolism by directly regulating carotenogenic genes. Plant Physiol. 2018;176(4):2657-76.

41. Ma N, Feng H, Meng X, Li D, Yang D, Wu C, et al. Overexpression of tomato SINAC1 transcription factor alters fruit pigmentation and softening. BMC Plant Biol. 2014;14(1):351

42. Zhu M, Chen G, Zhou S, Tu Y, Wang Y, Dong T, et al. A new tomato NAC (NAM/ATAF1/2/CUC2) transcription factor, SINAC4, functions as a positive regulator of fruit ripening and carotenoid accumulation. Plant Cell Physiol. 2014;55(1):119-35.

43. Sagawa JM, Stanley LE, LaFountain AM, Frank HA, Liu C, Yuan Y-W. An R2R3MYB transcription factor regulates carotenoid pigmentation in Mimulus lewisii flowers. New Phytol. 2016;209(3):1049-57.

44. Ampomah-Dwamena C, Thrimawithana AH, Dejnoprat S, Lewis D, Espley RV, Allan AC. A kiwifruit (Actinidia deliciosa) R2R3-MYB transcription factor modulates chlorophyll and carotenoid accumulation. New Phytol. 2019; 221(1):309-25.

45. Zhu F, Luo T, Liu C, Wang Y, Yang H, Yang W, et al. An R2R3-MYB transcription factor represses the transformation of $\alpha$ - and $\beta$-branch carotenoids by negatively regulating expression of $\mathrm{CrBCH} 2$ and $\mathrm{CrNCED} 5$ in flavedo of Citrus reticulate. New Phytol. 2017;216:178-92. 
46. Waters MT, Moylan EC, Langdale JA. GLK transcription factors regulate chloroplast development in a cell-autonomous manner. Plant J. 2010;56(3): 432-44.

47. Waters MT, Peng W, Muris K, Capper RG, Saunders NJ, Langdale JA. GLK transcription factors coordinate expression of the photosynthetic apparatus in Arabidopsis. Plant Cell. 2009;21(4):1109-28.

48. Powell ALT, Bennett AB. Uniform ripening encodes a Golden 2-like transcription factor regulating tomato fruit chloroplast development. Science. 2012;336(6089):1711-5.

49. Liang M-H, Jiang J-G. Analysis of carotenogenic genes promoters and WRKY transcription factors in response to salt stress in Dunaliella bardawil. Sci Rep. 2017;7(1):37025.

50. Han Y, Wu M, Cao L, Yuan W, Dong M, Wang X, et al. Characterization of OfWRKY3, a transcription factor that positively regulates the carotenoid cleavage dioxygenase gene OfCCD4 in Osmanthus fragrans. Plant Mol Biol. 2016;91(4):485-96.

51. García-Limones C, Schnäbele K, Blanco-Portales R, Luz Bellido M, Caballero $\mathrm{JL}$, Schwab W, et al. Functional characterization of FaCCD1: a carotenoid cleavage dioxygenase from strawberry involved in lutein degradation during fruit ripening. J Agric Food Chem. 2008;56(19):9277-85.

52. Brandi F, Bar E, Mourgues F, Horváth G, Turcsi E, Giuliano G, et al. Study of'Redhaven'peach and its white-fleshed mutant suggests a key role of CCD4 carotenoid dioxygenase in carotenoid and norisoprenoid volatile metabolism. BMC Plant Biol. 2011;11(1):24.

53. Rock CD, Zeevaart J. The ABA mutant of Arabidopsis thaliana is impaired in epoxy-carotenoid biosynthesis. Proc Natl Acad Sci U S A. 1991:88(17):7496-9.

54. Marin E, Nussaume L, Quesada A, Gonneau M, Sotta B, Hugueney P, et al. Molecular identification of zeaxanthin epoxidase of Nicotiana plumbaginifolia, a gene involved in abscisic acid biosynthesis and corresponding to the ABA locus of Arabidopsis thaliana. EMBO J. 1996; 15(10):2331-42.

55. Lee HS, Castle WS. Seasonal changes of carotenoid pigments and color in Hamlin, Earlygold, and budd blood orange juices. J Agric Food Chem. 2001; 49(2):877-82.

56. Grabherr MG, Haas BJ, Moran Y, Levin JZ, Thompson DA, Ido A, et al. Fulllength transcriptome assembly from RNA-Seq data without a reference genome. Nat Biotechnol. 2011;29(7):644

57. Pertea G, Huang X, Liang F, Antonescu V, Sultana R, Karamycheva S, et al. TIGR gene indices clustering tools (TGICL): a software system for fast clustering of large EST datasets. Bioinformatics. 2003;19(5):651-2.

58. Ana C, Stefan GT, Juan Miguel GG, Javier T, Manuel T, Montserrat R. Blast2GO: a universal tool for annotation, visualization and analysis in functional genomics research. Bioinformatics. 2005;21(18):3674-6.

59. Jia Y, Lin F, Hongkun Z, Yong Z, Jie C, Zengjin Z, et al. WEGO: a web tool for plotting GO annotations. Nucleic Acids Res. 2006;34:293-7.

60. Yeung KY, Ruzzo WL. Principal component analysis for clustering gene expression data. Bioinformatics. 2001;17(9):763-74.

61. Mortazavi A, Williams B, McCue K, Schaeffer L, Wold B. Mapping and quantifying mammalian transcriptomes by RNA-Seq. Nat Methods. 2008;5(7): $621-8$

62. Wang L, Feng Z, Wang X, Wang X, Zhang X. DEGseq: an R package for identifying differentially expressed genes from RNA-seq data. Bioinformatics. 2009;26(1):136-8.

63. Huang DW, Sherman BT, Lempicki RA. Systematic and integrative analysis of large gene lists using DAVID bioinformatics resources. Nat Protoc. 2009;4(1): 44.

64. Minoru K, Michihiro A, Susumu G, Masahiro H, Mika H, Masumi I, et al. KEGG for linking genomes to life and the environment. Nucleic Acids Res. 2008; 36(S1):D480-4.

65. Shannon P, Markiel A, Ozier O. Cytoscape: a software environment for integrated models of biomolecular interaction networks. Genome Res. 2003: 13(11):2498-504

66. Livak KJ, Schmittgen TD. Analysis of relative gene expression data using real-time quantitative $P C R$ and the $2-\Delta \Delta C T$ method. Methods. 2001;25(4) 402-8.

\section{Publisher's Note}

Springer Nature remains neutral with regard to jurisdictional claims in published maps and institutional affiliations.

Ready to submit your research? Choose BMC and benefit from:

- fast, convenient online submission

- thorough peer review by experienced researchers in your field

- rapid publication on acceptance

- support for research data, including large and complex data types

- gold Open Access which fosters wider collaboration and increased citations

- maximum visibility for your research: over $100 \mathrm{M}$ website views per year

At BMC, research is always in progress.

Learn more biomedcentral.com/submissions 